\title{
Andrea Schleu / Karin Schreiber-Willnow/Wolfgang Wöller (Hrsg.): Verwickeln und Entwickeln. Ethische Fragen in der Psychotherapie
}

\author{
Verlag für Akademische Schriften, 2014, Bad Homburg, \\ 148 Seiten, $14,80 €(D)$
}

Mit dieser Aufsatzsammlung möchten die HerausgeberInnen und AutorInnen eine Fehlerkultur etablieren, in der eine öffentliche Diskussion über Fehler, Misserfolge, Risiken oder Nebenwirkungen von Psychotherapien möglich wird. Dieses Ziel haben sie meines Erachtens erreicht. Von der Bedeutung der Ökonomisierung für helfende Berufe über ein Verständnis, wie es zu Verwicklungen und Entgleisungen in Therapien kommen kann, bis hin zu Hilfsangeboten bei solchen Therapien sowie Prävention und Aufklärung über Risiken und Nebenwirkungen von Psychotherapie erstreckt sich die Bandbreite der Beiträge.

Die meisten Aufsätze lesen sich kurzweilig und spannend - also keine trockene Kost mit erhobenem Zeigefinger. Stattdessen wird der Leser mit den alltäglichen Ambivalenzen und Dilemmata des therapeutischen Handelns konfrontiert und zum Nachdenken über die eigene Position angeregt. Die Quellen der Artikel sind vielfältig. Sie fußen unter anderem auf Forschung (zu Nebenwirkungen), auf Ergebnissen der Beschwerden und Beratungen eines Ethikvereins, auf stationärer klinischer Erfahrung (in der Konzentrativen Bewegungstherapie) sowie auf philosophischer Reflexion (zur Kunst des Verstehens, Würde und die Normalität von Fehlern).

Sehr erfrischend in diesem Buch ist die Lösungsorientierung. Häufig wird man als Leser frustriert, wenn durch die Darstellung einer misslichen Lage Problemfelder aufgetan werden und Hinweise zur Überwindung dieser Probleme ausbleiben. In dem vorliegenden Werk können TherapeutInnen sich auf Vorschläge sowohl für Haltungsänderungen, z. B. gleich zweifach in Hinblick auf die Ökonomisierung des Gesundheitswesens, als auch für konkrete Handlungsmöglichkeiten, z.B. bei sexuel- len Übergriffen in der Therapie, freuen. Es werden ethische Regeln und Normen grif-

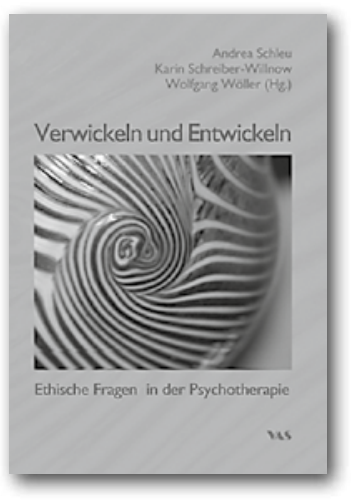

fig und übersichtlich definiert, so dass sich dieses kleine Buch zum schnellen Nachschlagen bei ethischen Unsicherheiten in der Praxis eignet. Die Auswirkungen von ethischen Regelverstößen werden erläutert, wodurch diese Regeln nicht als willkürliche Einschränkung wirken, sondern als in negativer Erfahrung begründete Schutzmaßnahmen verständlich werden.

Für Körper- und Bewegungspsychotherapeutlnnen von besonderem Interesse ist der Beitrag von Karin Schreiber-Willnow, die anhand von Fallbeispielen typische Risiken und Nebenwirkungen der Konzentrativen Bewegungstherapie im stationären Setting aufzeigt. Ihr Fazit sieht Fehler als Normalität und Chance für Wachstum in der Therapie. Somit ist es die Aufgabe von Körper- und BewegungstherapeutInnen, in Kenntnis und im Bewusstsein der Risiken Therapien zu leiten und „... das geeignete Maß an Belastung und Verwicklung für den Patienten zu finden, das für inn förderlich ist." (S.120)

Die Thematisierung von Fehlern sollte bereits in der Ausbildung von TherapeutInnen geschehen. Hierzu wäre dieses Buch ein hervorragenderer Ausgangspunkt. Für bereits praktizierende TherapeutInnen und Supervisorlnnen bietet das Werk eine Reflexionshilfe, eine Quelle von Lösungen für Problemfälle und schließlich, im offenen Umgang damit, dass alle Menschen und alle therapeutischen Verfahren fehlerhaft sind, die Grundlage für eine Fehlerkultur.

\section{Dr. Marianne Eberhard-Kaechele}

DOI 10.2378/ ktb2016.art26d 